\title{
Correction to: Use of multicolor fluorescence in situ hybridization to detect deletions in clinical tissue sections
}

\author{
Maisa Yoshimoto ${ }^{1}$ - Olga Ludkovski ${ }^{2}$. Jennifer Good ${ }^{3}$. Ciro Pereira ${ }^{4}$ - Robert J. Gooding ${ }^{5}$. \\ Jean McGowan-Jordan ${ }^{6,7} \cdot$ Alexander Boag $^{3} \cdot$ Andrew Evans ${ }^{2} \cdot$ Ming-Sound Tsao $^{2} \cdot$ Paulo Nuin $^{1}$. \\ Jeremy A. Squire $\mathbb{I}^{1,3,4,8}$
}

(c) United States \& Canadian Academy of Pathology 2018

Correction to: Laboratory Investigation; https://doi.org/10. 1038/s41374-017-0007-2; Article published online 16 Jan 2018.
Figure 2 is incorrect in the original version of this article. The correct figure 2 is provided below

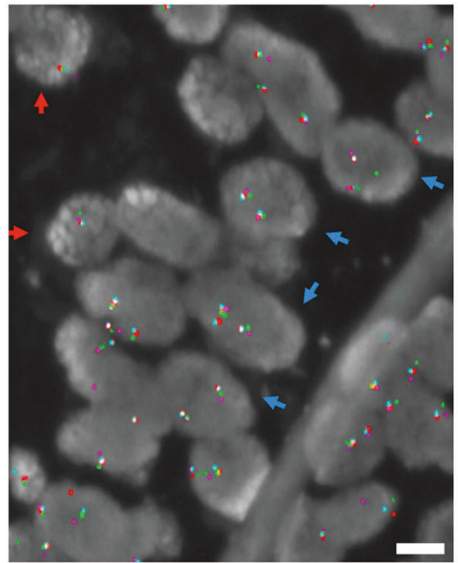

A

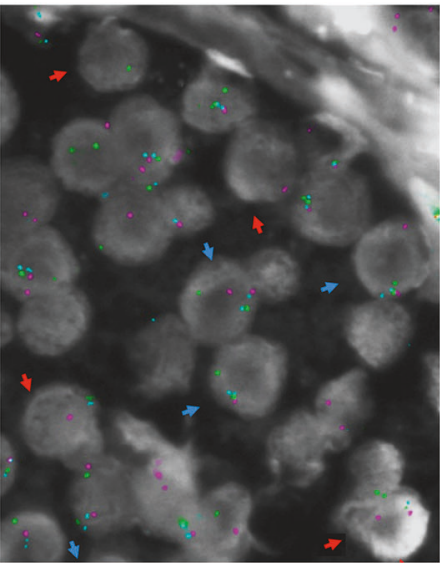

B

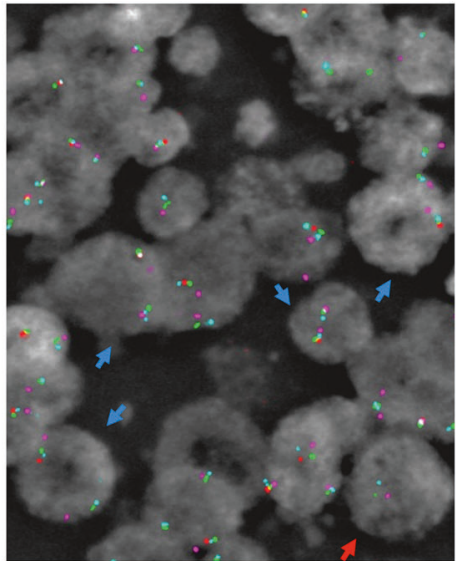

C

The original article can be found online at https://doi.org/10.1038/ s41374-017-0007-2.

Jeremy A. Squire

jsquireinsp@gmail.com

1 Department of Medical Genetics, University of Alberta, Edmonton, AB, Canada

2 Department of Pathology, University Health Network, Toronto, ON, Canada

3 Department of Pathology and Molecular Medicine, Queen's University, Kingston, ON, Canada

4 Departments of Genetics, School of Medicine of Ribeirão Preto,
University of Sao Paulo, São Paulo, Brazil

5 Department of Physics Engineering Physics and Astronomy, Queen's University, Kingston, ON, Canada

6 Genetics Diagnostic Laboratory, Children's Hospital of Eastern Ontario, Ottawa, ON Canada

7 Department of Pathology and Laboratory Medicine, University of Ottawa, Ottawa ON Canada

8 Department of Pathology and Legal Medicine School of Medicine of Ribeirão Preto, University of São Paulo, São Paulo, Brazil 